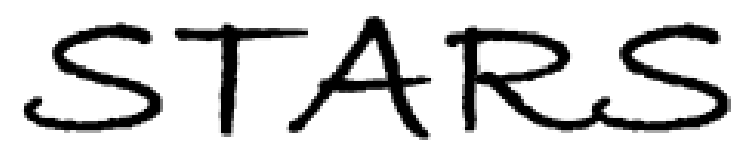

University of Central Florida

STARS

$1-1-2012$

\title{
Characterization of Active Main Belt Object P/2012 F5 (Gibbs): A Possible Impacted Asteroid
}

\author{
Rachel Stevenson \\ University of Central Florida \\ E. A. Kramer \\ University of Central Florida \\ J. M. Bauer \\ University of Central Florida \\ J. R. Masiero \\ University of Central Florida
}

\section{A. K. Mainzer \\ University of Central Florida}

Find similar works at: https://stars.library.ucf.edu/facultybib2010

University of Central Florida Libraries http://library.ucf.edu

This Article is brought to you for free and open access by the Faculty Bibliography at STARS. It has been accepted for inclusion in Faculty Bibliography 2010 s by an authorized administrator of STARS. For more information, please contactSTARS@ucf.edu.

\section{Recommended Citation}

Stevenson, Rachel; Kramer, E. A.; Bauer, J. M.; Masiero, J. R.; and Mainzer, A. K., "Characterization of Active Main Belt Object P/2012 F5 (Gibbs): A Possible Impacted Asteroid" (2012). Faculty Bibliography 2010s. 3353.

https://stars.library.ucf.edu/facultybib2010/3353

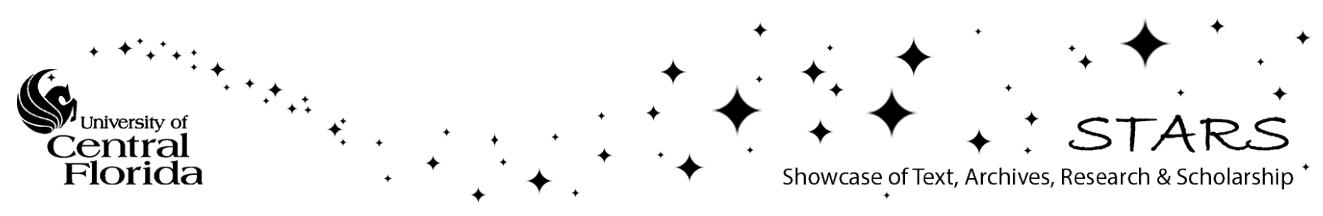




\title{
CHARACTERIZATION OF ACTIVE MAIN BELT OBJECT P/2012 F5 (GIBBS): A POSSIBLE IMPACTED ASTEROID
}

\author{
R. Stevenson ${ }^{1}$, E. A. Kramer ${ }^{1,2}$, J. M. Bauer ${ }^{1}$, J. R. Masiero ${ }^{1}$, and A. K. Mainzer ${ }^{1}$ \\ ${ }^{1}$ Jet Propulsion Laboratory, Caltech, 4800 Oak Grove Drive, Pasadena, CA 91109, USA; Rachel.A.Stevenson@jpl.nasa.gov \\ ${ }^{2}$ Department of Physics, University of Central Florida, 4000 Central Florida Blvd. Orlando, FL 32816, USA \\ Received 2012 May 11; accepted 2012 September 24; published 2012 October 29
}

\begin{abstract}
In this work, we characterize the recently discovered active main belt object P/2012 F5 (Gibbs), which was discovered with a dust trail $>7$ in length in the outer main belt, 7 months prior to aphelion. We use optical imaging obtained on UT 2012 March 27 to analyze the central condensation and the long trail. We find $B$-band and $R$-band apparent magnitudes of $20.96 \pm 0.04$ mag and $19.93 \pm 0.02 \mathrm{mag}$, respectively, which give an upper limit on the radius of the nucleus of $2.1 \mathrm{~km}$. The geometric scattering cross-section of material in the trail was $\sim 4 \times 10^{8} \mathrm{~m}^{2}$, corresponding to a mass of $\sim 5 \times 10^{7} \mathrm{~kg}$. Analysis of infrared images taken by the Wide-field Infrared Survey Explorer in 2010 September reveals that the object was below the detection limit, suggesting that it was less active than it was during 2012, or possibly inactive, just six months after it passed through perihelion. We set a $1 \sigma$ upper limit on its radius during this time of $2.9 \mathrm{~km}$. P/2012 F5 (Gibbs) is dynamically stable in the outer main belt on timescales of $\sim 1 \mathrm{Gyr}$, pointing toward an asteroidal origin. We find that the morphology of the ejected dust is consistent with it being produced by a single event that occurred on UT 2011 July $7 \pm 20$ days, possibly as the result of a collision with a small impactor.
\end{abstract}

Key words: comets: individual (P/2012 F5 (Gibbs)) - minor planets, asteroids: individual (P/2012 F5)

Online-only material: color figures

\section{INTRODUCTION}

In recent years, a new class of objects with asteroid-like orbits but comet-like behavior has been identified within the main belt. These objects occupy low-eccentricity, low-inclination orbits with Tisserand parameters $\left(T_{J}\right)>3$, placing them squarely in the asteroid regime. However, they exhibit mass loss in a manner more akin to those observed around comets. Though only a handful of these objects are known at this time, they cover a wide range of orbital space $(2.29 \mathrm{AU}<a<3.20 \mathrm{AU}$, $0.12<e<0.34,0.2<i<21.4$, where $a, e$, and $i$ represent semimajor axis, eccentricity, and inclination, respectively), their sizes vary by several orders of magnitude (Bauer et al. 2012), and the morphologies observed include spherical comae, dust tails, and/or persistent debris trails. The scientific community has yet to agree on a name for such objects. For this work, we find that the terms "active asteroid" (Jewitt 2012), "activated asteroid" (Licandro \& Campins 2010), "active main belt object" (Bauer et al. 2012), and the original definition of "main belt comet" (Hsieh \& Jewitt 2006) all apply. Potentially, depending on the driver of the observed activity, either the refined definition of main belt comet or "disrupted asteroid" (Hsieh et al. 2012a) may also apply. For simplicity, in this work we choose to utilize the term "active main belt object" (AMBO), which encompasses all objects with comet-like morphologies and main belt asteroidal orbits, regardless of the suspected driver of activity.

Studies have shown that most of the known AMBOs are dynamically stable on timescales longer than $100 \mathrm{Myr}$ (e.g., Hsieh et al. 2012a, 2012b), suggesting they are long-term residents of the main belt. Conversely, 238P and P/2008 R1 are stable for only $\sim 20-30 \mathrm{Myr}$, respectively, and thus may be interlopers from elsewhere in the solar system (Haghighipour 2009; Jewitt et al. 2009).

One of the best-characterized AMBOs, 133P/Elst-Pizzaro, has been active at multiple epochs, leading to the suggestion that the activity was driven by seasonal heating of an active area (Elst et al. 1996; Hsieh et al. 2004, 2010). A second AMBO, 238P/Read has also shown repeated activity, lending credence to the sublimation hypothesis (Hsieh et al. 2011). Their behavior mimics that of dynamical comets, which become active within a few AU of the Sun as volatile deposits are heated. Work by Schorghofer (2008) suggests that sub-surface ice deposits could survive in the main belt for billions of years at depths of just a few meters. Spectroscopic searches for gas emission lines have, to date, been unsuccessful, but this may stem from the weakness of the outgassing (e.g., Jewitt et al. 2009; Licandro et al. 2011; Hsieh et al. 2012a). Observations of 596 (Scheila) and P/2010 A2 (LINEAR) did not appear to fit with the model of mass loss driven by prolonged sublimation. Instead, these asteroids displayed morphologies better explained by impulsive mass-loss events, consistent with collisions (Jewitt et al. 2010, 2011; Snodgrass et al. 2010).

On UT 2012 March 22.89, A. R. Gibbs reported the discovery of a new comet that appeared with a narrow dust trail greater than $7^{\prime}$ in length. Subsequent observations confirmed the discovery and the apparent morphology (Gibbs et al. 2012). The object was given the cometary designation P/2012 F5 (Gibbs), hereafter referred to as P/2012 F5. On UT 2012 June 21, ephemerides from the JPL HORIZONS System ${ }^{3}$ resulted in orbital elements of $a=3.0038 \mathrm{AU}, e=0.042$, and $i=9.739$ (Table 1), an orbital period of $5.21 \mathrm{yr}$, and a Tisserand parameter of $T_{J}=3.23$. The corresponding $1 \sigma$ uncertainties are $6.3 \times 10^{-5} \mathrm{AU}, 8.4 \times 10^{-5}$, and $4.1 \times 10^{-4}$ for $a, e$, and $i$, respectively. The comet-like appearance but asteroid-like orbit of P/2012 F5 make it the ninth known AMBO.

In this work, we characterize $\mathrm{P} / 2012 \mathrm{~F} 5$ using optical imaging data obtained at Palomar Observatory just three days after its discovery. We also use archived infrared images taken by the

\footnotetext{
3 http://ssd.jpl.nasa.gov/?horizons
} 
Table 1

Orbital Elements of P/2012 F5

\begin{tabular}{lccccccc}
\hline \hline Epoch & $\begin{array}{c}a^{\mathrm{a}} \\
(\mathrm{AU})\end{array}$ & $\begin{array}{c}q^{\mathrm{b}} \\
(\mathrm{AU})\end{array}$ & $e^{\mathrm{c}}$ & $\begin{array}{c}i^{\mathrm{d}} \\
(\mathrm{deg})\end{array}$ & $\begin{array}{c}\Omega^{\mathrm{e}} \\
(\mathrm{deg})\end{array}$ & $\begin{array}{c}\omega^{\mathrm{f}} \\
(\mathrm{deg})\end{array}$ & $\begin{array}{c}M^{\mathrm{g}} \\
(\mathrm{deg})\end{array}$ \\
\hline 2012 Mar. 28.0 & 3.00 & 2.88 & 0.04 & 9.74 & 216.9 & 177.4 & 138.4 \\
\hline
\end{tabular}

Notes.

a Semimajor axis.

b Perihelion.

c Eccentricity.

d Inclination.

e Longitude of the ascending node.

${ }^{\mathrm{f}}$ Argument of perihelion.

g Mean anomaly.

Wide-field Infrared Survey Explorer (WISE) in 2010 September when the comet was six months post-perihelion. Through aperture photometry, we constrain the size of the nucleus and estimate the mass of dust within the trail. We use the morphology and dynamical modeling of the trail to characterize the duration and onset time of the activity, as well as the properties of the dust grains emitted. Finally, the dynamical stability of P/2012 F5 is investigated through the use of a symplectic integrator. Our results are summarized in the conclusions.

\section{OBSERVATIONS AND DATA REDUCTION}

In this paper, we use optical ground-based observations and infrared space-based observations to characterize P/2012 F5. Table 2 provides a summary of observations.

\subsection{Large Format Camera, Palomar Observatory}

We observed P/2012 F5 with the Large Format Camera (Simcoe et al. 2000) mounted on the 200" Hale telescope atop Mount Palomar on UT 2012 March 27. The array of six $2048 \times 4096$ pixel CCDs provided a $24^{\prime}$ on a side field of view, with a resolution of 0 '. 35 pixel $^{-1}$ when using $2 \times 2$ binning. A dithering pattern was invoked to provide coverage in the $\sim 15^{\prime \prime}$ gaps between the chips. We used $B$ and $R$ Bessel filters with central wavelengths and bandwidths of $4400 \AA$ and $1000 \AA$, and $6300 \AA$ and $1200 \AA$, respectively. Two 180 s exposures and five $90 \mathrm{~s}$ exposures were obtained with the $B$ and $R$ Bessel filters, respectively. The object was tracked at non-sidereal rates as given by the JPL Horizons ephemeris.

The images were debiased and flattened using bias and twilight flat-field frames obtained on the night of the observations. Amplifier glow impacted all of the images in the southernmost chip. We investigated the extent of the contamination by comparing the median of a $\sim 114^{\prime \prime} \times 20^{\prime \prime}$ box near the area of
P/2012 F5's trail closest to the region of image most affected by the amplifier glow to the median of a background region far from both the amplifier and P/2012 F5. We found that the amplifier glow had no effect on the $R$-band photometry, but that the amplifier glow contributed $\sim 0.03$ mag to the background flux at the northwestern end of the trail in the longer exposures obtained in $B$ band.

Photometric calibration was done using Landolt standards (Landolt 1992). Photometric uncertainties, including photon statistics, were found to be less than $0.05 \mathrm{mag}$. The debiased, flattened images were median combined to produce a deeper single image in each filter. The stellar point spread function (PSF) FWHM was on the order of 1".7 for the individual images.

The observations were obtained when P/2012 F5 was approximately seven months pre-aphelion (true anomaly $=141^{\circ}$ ) at a heliocentric distance of $3.10 \mathrm{AU}$. The object appeared as a bright yet unresolved condensation in the southeast corner of the image with a long trail (Figure 1).

\subsection{Wide-field Infrared Survey Explorer}

We used archived data from the $40 \mathrm{~cm}$ WISE telescope to search pre-discovery images for signs of P/2012 F5. The data were obtained between UT 2010 September 22 and 24 after the cryogen was depleted in the secondary tank. Consequently, only data in the three shortest wavelength bands (W1: $3.4 \mu \mathrm{m}$, W2: $4.6 \mu \mathrm{m}$, and W3: $12 \mu \mathrm{m}$ ) were available. The WISE field of view is $47^{\prime} \times 47^{\prime}$ with a pixel scale of $2^{\prime \prime} .75$ pixel $^{-1}$. Simultaneous exposures were taken in each band every $11 \mathrm{~s}$, with nominal exposure times of $7.7 \mathrm{~s}$ in W1 and W2, and $1.1 \mathrm{~s}$ in W3 (Wright et al. 2010; Mainzer et al. 2011a).

Instrumental, photometric, and astrometric calibrations were performed by the "first pass" scan/frame pipeline (Cutri et al. 2011). The apparent velocity of $\mathrm{P} / 2012 \mathrm{~F} 5$ at the time of observation was $31^{\prime \prime} \mathrm{hr}^{-1}$, corresponding to a drift across the frame of less than $0{ }^{\prime} 01$ during the integrations. Since this is far smaller than the pixel scale of each image, the effect of trailing was negligible.

We calculated the positional uncertainty of P/2012 F5 using information retrieved from the JPL Horizons Ephemeris service on UT 2012 June 21 . The $3 \sigma$ positional uncertainty was 2'.5. We thus rejected any frame in which the predicted position was less than $5^{\prime}$ from the edge of the field of view. This resulted in a total of 11 useful scans of the prediscovery field. The individual images in each band were shifted to compensate for the motion of P/2012 F5 and were co-added using the "A WISE Astronomical Image Co-adder" algorithm (Masci \& Fowler 2009), resulting in resampled images with pixel scales of $1^{\prime \prime} .0$ pixel $^{-1}$. The FWHM of the stacked images were 6.'1,

Table 2

Journal of Observations

\begin{tabular}{|c|c|c|c|c|c|c|c|c|}
\hline $\begin{array}{l}\text { Date } \\
\text { (UT) }\end{array}$ & Observatory & Filter & $N^{\mathrm{a}}$ & $\begin{array}{c}\text { Exposure Time } \\
\text { (s) }\end{array}$ & $\begin{array}{l}r_{H}^{\mathrm{b}} \\
(\mathrm{AU})\end{array}$ & $\begin{array}{c}\Delta^{\mathrm{c}} \\
(\mathrm{AU})\end{array}$ & $\begin{array}{c}\alpha^{\mathrm{d}} \\
(\mathrm{deg})\end{array}$ & $\begin{array}{l}\text { Image Scale } \\
\left(\mathrm{km} \mathrm{pixel}^{-1}\right)\end{array}$ \\
\hline 2010 Sep. 22-24 & WISE & W3 & 11 & 1.1 & 2.90 & 2.7 & 20.2 & 5400 \\
\hline 2012 Mar. 27.3 & Palomar & $R$ & 5 & 90 & 3.10 & 2.1 & 5.8 & 535 \\
\hline 2012 Mar. 27.3 & Palomar & $B$ & 3 & 180 & 3.10 & 2.1 & 5.8 & 535 \\
\hline
\end{tabular}

Notes.

a Number of exposures.

b Heliocentric distance.

c Geocentric distance.

${ }^{\mathrm{d}}$ Phase angle. 


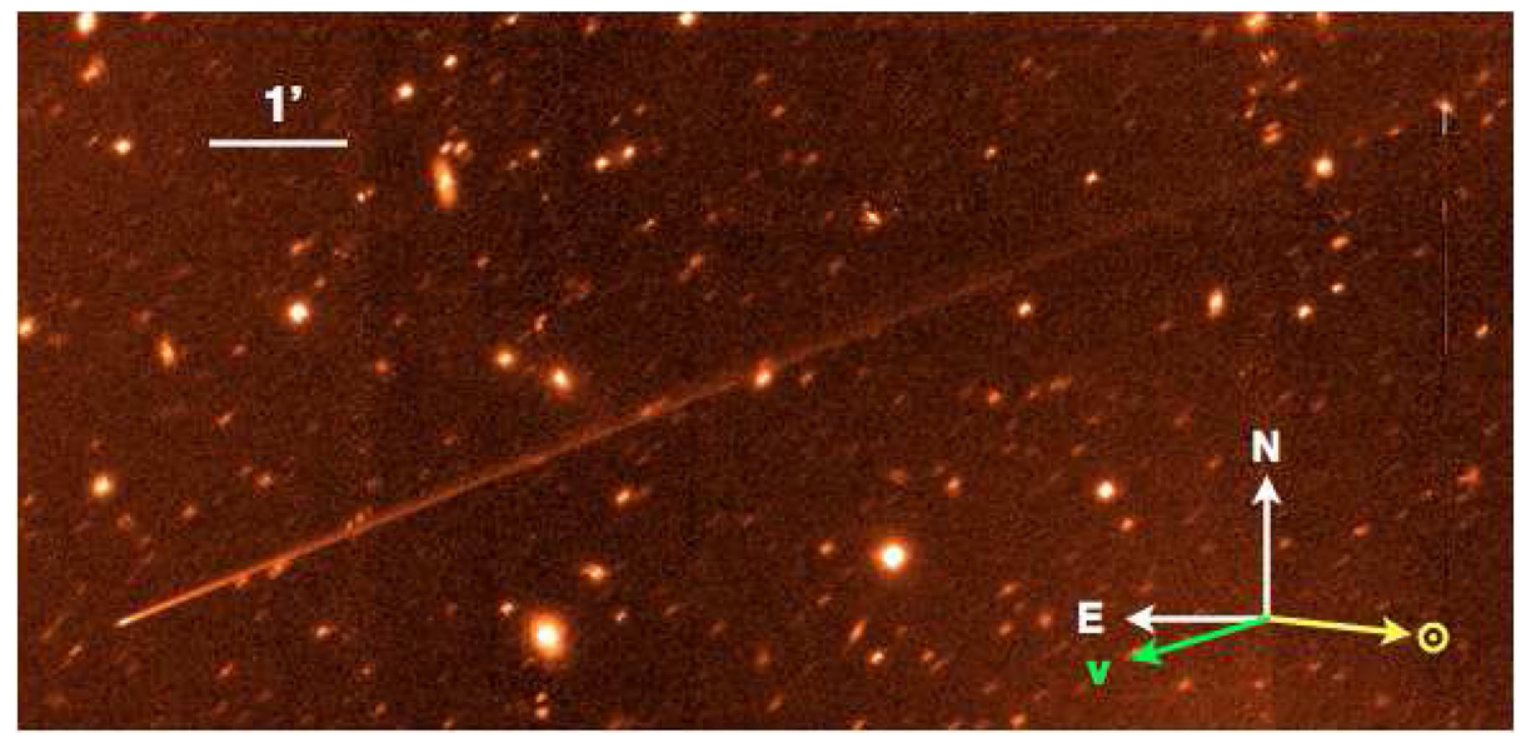

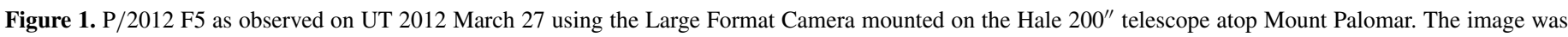

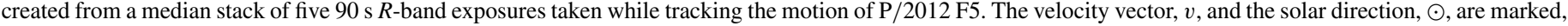
as are the directions north and east.

(A color version of this figure is available in the online journal.)

6."4, and 6.5 in bands W1, W2, and W3, respectively (Wright et al. 2010; Mainzer et al. 2011a). The $3 \sigma$ uncertainty associated with the on-sky velocity of P/2012 F5 was small enough that the resulting deviation from its predicted position was several times smaller than the FWHM of each image.

The observation dates of the pre-discovery fields correspond to six months post-perihelion (true anomaly $\sim 38^{\circ}$ ) when $\mathrm{P} / 2012 \mathrm{~F} 5$ was at a heliocentric distance of $2.90 \mathrm{AU}$.

\section{RESULTS}

\subsection{Nucleus Photometry at Optical Wavelengths}

Though the nucleus of P/2012 F5 is obscured by a dust coma, a bright condensation exists at the leading end of the trail. We use aperture photometry to constrain its brightness with the goal of characterizing the source of the ejecta. The aperture radius was selected to be $\sim 2.5$ times larger than the FWHM of the images but small enough to focus on the material closest to the nucleus. The aperture was centered on the optocenter of the condensation. We note that the aperture contains non-negligible contamination from the coma and dust trail, and present the resulting cross-sections and radii as upper limits on the nucleus only. Using a circular aperture with a radius of 2 ". 1 $(3260 \mathrm{~km}$ as projected on the sky at the heliocentric distance of P/2012 F5 at the time of observation), we calculate apparent $B$-band and $R$-band magnitudes of $m_{B}=20.96 \pm 0.04$ mag and $m_{R}=19.93 \pm 0.02 \mathrm{mag}$, respectively. The $B-R$ color of the near-nucleus region is $1.03 \pm 0.04 \mathrm{mag}$, which is consistent with solar colors.

We correct for the observing geometry by converting the apparent magnitudes, $m_{B}, m_{R}$, to absolute magnitudes, $H_{B}$, $H_{R}$, assuming a phase coefficient, $\beta_{\alpha}$, of $0.04 \mathrm{mag} \mathrm{deg}^{-1}$, as is common for both active and inactive comets (Lamy et al. 2004), as well as C-type asteroids, which dominate the outer main belt (Belskaya \& Shevchenko 2000). We calculate a phase correction of $0.23 \mathrm{mag}$ for the data obtained at Palomar. The corresponding absolute magnitudes in $B$ band and $R$ band, respectively, are $16.66 \pm 0.04 \mathrm{mag}$ and $15.63 \pm 0.02 \mathrm{mag}$. The concept of absolute magnitude is not strictly valid for extended objects, given that an aperture of fixed angular size will include varying amounts of coma when the comet is observed at different heliocentric distances.

\subsection{Photometry of the Trail}

We rotate the stacked images of $\mathrm{P} / 2012 \mathrm{~F} 5$ so that the trail is aligned horizontally with the image axis with the head of the trail to the left. We place a rectangular aperture around the trail, using the visible extent of the trail to set its boundaries. The trail is not symmetrical along its breadth and extends farther northeast than it does southwest. The photometric aperture, therefore, is set to extend $8^{\prime \prime} .75\left(1.4 \times 10^{4} \mathrm{~km}\right.$ as projected on the sky) in the northeast direction, and $5.25\left(8.1 \times 10^{3} \mathrm{~km}\right.$ projected distance $)$ to the southwest and centered on the opto-center of the trail along its length. The aperture is then divided into 144 ( $B$ band) or 148 ( $R$ band) segments along the length of the trail, each having dimensions of $3^{\prime \prime} .5 \times 14^{\prime \prime} .0$. The aperture used in $B$ band had a total size of $504^{\prime \prime} \times 14^{\prime \prime}$, while the $R$-band aperture had a size of $518^{\prime \prime} \times 14^{\prime \prime}$, corresponding to physical distances of $7.8 \times$ $10^{5} \mathrm{~km}$ by $2.2 \times 10^{4} \mathrm{~km}$, and $8.0 \times 10^{5} \mathrm{~km}$ by $2.2 \times 10^{4} \mathrm{~km}$ at the heliocentric distance of $\mathrm{P} / 2012 \mathrm{~F} 5$ at the time of observation. Due to the large size of the trail and relatively small motion of P/2012 F5, numerous background objects contaminate the aperture. We digitally remove the four worst offenders from the stacked $B$-band image, and the six worst from the stacked $R$-band image using the IRAF task IMEDIT, which replaces marked pixels with values interpolated from nearby sky regions. We conservatively estimate that background objects remaining in the aperture contribute an uncertainty of $\sim 0.2 \mathrm{mag}$. The conversion of photometric magnitudes to physical properties, such as mass of the dust, are strongly dependent on the assumed properties of the dust, many of which, such as particle size distribution, reflectivity, and density, are uncertain by more than $20 \%$. We therefore consider the intrusion of background objects an additional source of error that is of order or less than other sources. In order to estimate the sky background along the trail, we use a similar method to Hsieh et al. $(2004,2010)$ and 

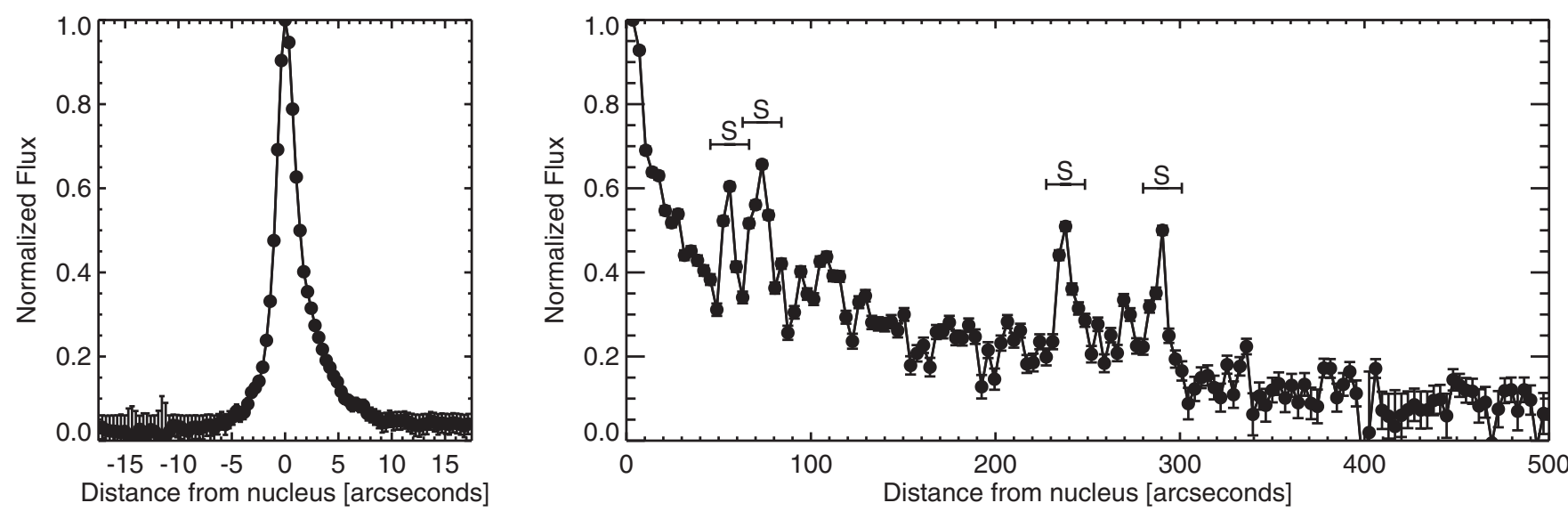

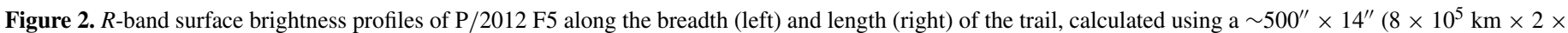

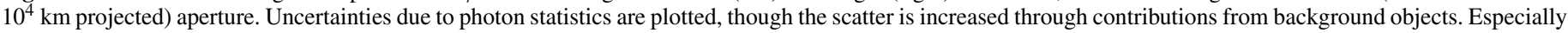
bright background stars are labeled with "S."

place rectangular sky background apertures of 3".5 $\times 2$ 2.. $8(5.4 \times$ $10^{3} \mathrm{~km}$ by $4.3 \times 10^{3} \mathrm{~km}$ projected) directly above and below the apertures along the trail. The sky background is computed as the median of the pixels within these sky apertures and is subtracted from each pixel within the box apertures placed along the trail.

Figure 2 shows the normalized brightness profiles of the trail, as measured along its breadth and length. The crosssection through the trail's breadth is noticeably asymmetric, with the northeastern edge (positive distance from the nucleus in Figure 2) being more diffuse than the sharper southwestern edge. We note that many cometary dust tails and trails show asymmetries, perhaps most noticeably the trail of P/2010 A2 (LINEAR), which retained an unusual and highly asymmetric morphology for nine months after undergoing an outburst in the main belt (Jewitt et al. 2010; Moreno et al. 2010; Snodgrass et al. 2010). Thus, this asymmetry may be a result of the intrinsic structure present in the ejected material shortly after the outburst. The surface brightness decreases along the length of the trail with increasing distance from the nucleus, $d$, approximately as $d^{-0.4}$. This is a somewhat shallower decrease than the relationship of $d^{-0.6}$ that was found for 133P/Elst-Pizarro as observed in 2002 by Hsieh et al. (2004). The material within the trail, as measured using the rectangular apertures previously described, has total magnitudes of $17.41 \pm 0.20 \mathrm{mag}$ and $16.25 \pm 0.20 \mathrm{mag}$ in $B$ and $R$ bands, respectively. We use 3." $5 \times 14^{\prime \prime} .0$ segments to measure the $B-R$ color along the trail between the nucleocentric distances of $14^{\prime \prime}$ and $504^{\prime \prime}$. We find the median color of the segments to be $1.12 \pm 0.28 \mathrm{mag}$, consistent with both the color of the region at the head of the trail, and solar colors. We note that the large uncertainty also renders it consistent with the color of dust around active comets ( $B-R \sim 1.0-1.4$ mag; Jewitt \& Meech 1986; Kolokolova et al. 2004).

\subsection{Constraints From WISE Infrared Photometry}

Using the stacked WISE images, an area of $600^{\prime \prime} \times 600^{\prime \prime}$ was searched for any PSF-like signal that exceeded a $1 \sigma$ limit above the sky background using a 9'.0 radius aperture $(1.8 \times$ $10^{4} \mathrm{~km}$ projected distance). $\mathrm{P} / 2012 \mathrm{~F} 5$ was not detected in any wavelength band by the WISE mission. By characterizing the sky background in W3 (the most sensitive band to thermal dust emission), we set a $3 \sigma$ detection limit on any potential objects within the field of $5 \mathrm{mJy}$, which corresponds to a lower limit on the absolute magnitude of material within the aperture of $15.2 \mathrm{mag}$.

\section{ANALYSIS}

\subsection{Dust Morphology}

The standard method for understanding the morphology of cometary dust tails and trails is the Finson-Probstein model (Finson \& Probstein 1968). This model assumes that once cometary dust particles leave the surface, their motion is governed by two forces: solar gravity and solar radiation pressure. The particle motion can then be parameterized using the ratio, $\beta$ of these two forces:

$$
\beta=\frac{F_{\text {rad }}}{F_{\text {grav }}} .
$$

In physical units, this gives the ratio:

$$
\beta=\frac{1.19 \times 10^{-3} Q_{\mathrm{pr}}}{\rho_{d} d},
$$

where $Q_{\mathrm{pr}}$ is the scattering efficiency for radiation pressure, $\rho_{d}$ is the bulk density of the particle, $d$ is the particle diameter, and the factor of $1.19 \times 10^{-3}\left(\mathrm{~kg} \mathrm{~m}^{-2}\right)$ comes from multiplying all the constant values (Finson \& Probstein 1968). For grain sizes of $d>\lambda, Q_{\mathrm{pr}} \sim 1$ (Burns et al. 1979); given the central wavelengths of the filters used, this condition holds for $d \gtrsim$ $0.5 \mu \mathrm{m}$.

$\beta$ is incorporated into the equation of motion in the following way:

$$
\ddot{\boldsymbol{x}}+(1-\beta) \frac{G M_{\odot}}{|\boldsymbol{x}|^{3}} \boldsymbol{x}=0
$$

where $G$ is the universal gravitational constant, $M_{\odot}$ is the mass of the Sun, and $\boldsymbol{x}$ is the vector position of the object. This is a simple equation of motion that can then be integrated for different values of $\beta$ to track the motion of particles with a particular $\beta$ value.

The computations were carried out by creating a numerical integrator (based on the work of Lisse et al. 1998) in the language Python which took in a set of $\beta$ values $(0.0001<\beta<$ 3.0000), and integrated the motion of the dust particles over the designated time interval. This generated a set of points which 

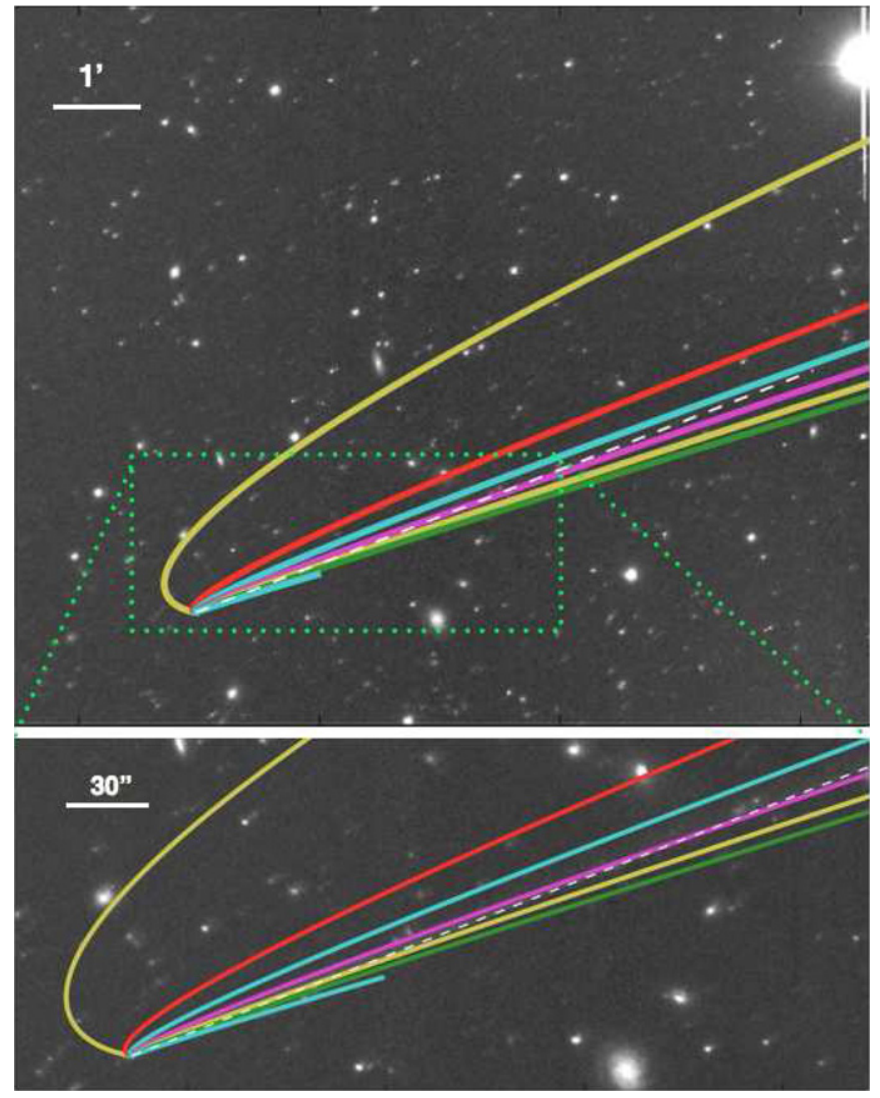

Figure 3. Syndynes tracing particles with $\beta=0.0003-3.0$ (bottom to top, cyan through yellow) are plotted over a stacked $R$-band image of P/2012 F5 from UT 2012 March 27. The syndynes do not recreate the observed morphology and diverge from the trail (highlighted by the white dashed line) at large nucleocentric distances. The best-fitting syndyne $(\beta=0.03$, magenta) initially curves north of the trail then crosses to the southwest approximately 200" $\left(3.1 \times 10^{5} \mathrm{~km}\right.$ projected $)$ from the nucleus. We find that synchrones provide a much better fit, suggesting the dust was released during a single event, rather than over a prolonged period.

(A color version of this figure is available in the online journal.)

can be shown as curves of particles with constant $\beta$ released at a range of times (syndynes) or curves of constant release date with a range of particle sizes (synchrones).

Figure 3 shows plots of the syndyne models plotted on top of the $R$-band data. At first look, the $\beta=0.03$ (shown in magenta) syndyne seems to model the trail (highlighted with a white dashed line) well. However, upon closer inspection it is clear that this syndyne initially is northeast of the trail, then curves southwest and crosses the trail approximately 200" (projected distance: $3.1 \times 10^{5} \mathrm{~km}$ ) from the nucleus.

When the model is instead shown as synchrones (curves of constant particle release date; Figure 4), the fit is greatly improved. The particle emission date was constrained by modeling the width of the trail with a Gaussian, which yielded a half-width half-maximum of about $1^{\prime \prime} .05$. We then calculated where the $\beta=$ 0.03 syndyne came within this distance from, and then crossed, the center of the trail. This revealed a best-fit synchrone of $264 \pm 20$ days, as shown as a green line in Figure 4. This corresponds to a particle ejection date of UT 2011 July $7 \pm 20$ days when P/2012 F5 was at a heliocentric distance of 3.01 AU and a true anomaly of $\sim 94^{\circ}$.

We use the observed length of the trail to constrain the size of the particles, given that the distances traveled by dust grains since the ejection event are size-dependent. We find that particles

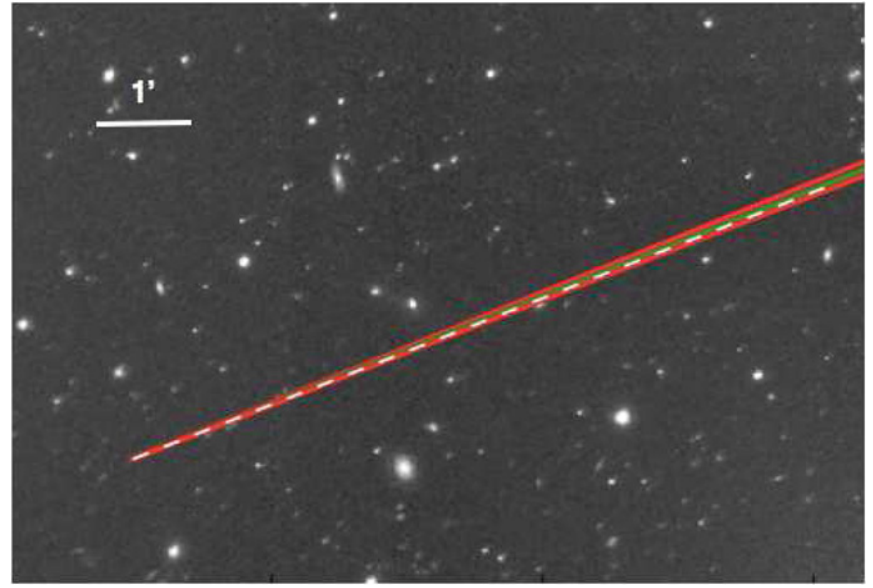

Figure 4. Best-fit synchrone (green) is plotted on the stacked $R$-band image with additional synchrones representing the uncertainties (red). The position and approximate length of the trail is marked by the dashed white line. Particles represented by the best-fit synchrone must have been released $264 \pm 20$ days prior to the observation, which corresponds to an ejection date near UT 2011 July 7.

(A color version of this figure is available in the online journal.)

with radii of $\sim 20 \mu \mathrm{m}$ could have traveled $\sim 8 \times 10^{5} \mathrm{~km}$ to the end of the observed trail in 264 days, setting a lower limit on the size of particles present. Smaller particles could have traveled further but are not observed in our data, possibly because they were either not originally released or had a scattering crosssection that fell below our detection limit.

\subsection{Physical Characterization of $P / 2012 F 5$ and its Activity}

The results from the aperture photometry can be used to constrain the physical properties of the nucleus and adjacent coma. We use Equation (4) to estimate the geometric crosssection of material, $\sigma\left(\mathrm{m}^{2}\right)$, within the aperture:

$$
p_{\lambda} \Phi_{\alpha} \sigma=2.24 \times 10^{22} \pi r_{H}^{2} \Delta^{2} 10^{0.4\left(m_{\odot \lambda}-m_{\lambda}\right)},
$$

where $p_{\lambda}$ is the geometric albedo, $m_{\odot \lambda}$ is the apparent magnitude of the Sun, and $m_{\lambda}$ is the apparent magnitude of the material within the aperture, each given for a specific broadband filter (Jewitt 1991). We assume the albedo of material is 0.04 in both $B$ and $R$ bands, which is broadly consistent with observations of AMBOs, outer main belt asteroids, and comets (e.g., Jewitt \& Meech 1986; Lamy et al. 2004; Hsieh et al. 2009a; Masiero et al. 2011; Bauer et al. 2012). The apparent magnitudes of the Sun in $B$ and $R$ bands are -26.10 and -27.12 , respectively. ${ }^{4}$ The parameter $\Phi_{\alpha}$ is a function to correct for phase-angle-dependent variations in brightness and is calculated using Equation (5):

$$
-2.5 \log _{10} \Phi_{\alpha}=\alpha \beta_{\alpha},
$$

where $\alpha(\mathrm{deg})$ is the phase angle and $\beta_{\alpha}\left(\mathrm{mag} \mathrm{deg}^{-1}\right)$ is the phase coefficient, already defined as having a value of $0.04 \mathrm{mag} \mathrm{deg}^{-1}$.

Considering the apparent magnitudes of material within a 2 ". 1 aperture, we estimate the geometric cross-section of the material to be $\sim 1.4 \times 10^{7} \mathrm{~m}^{2}$ in $B$ band and $\sim 1.5 \times 10^{7} \mathrm{~m}^{2}$ in $R$ band. If the light was being reflected by an inactive, spherical body, the effective radius of the object would be $\sim 2.1 \mathrm{~km}$. This sets an upper limit on the size of $\mathrm{P} / 2012$ F5, though, given the extensive

\footnotetext{
4 http://mips.as.arizona.edu/ cnaw/sun.html
} 

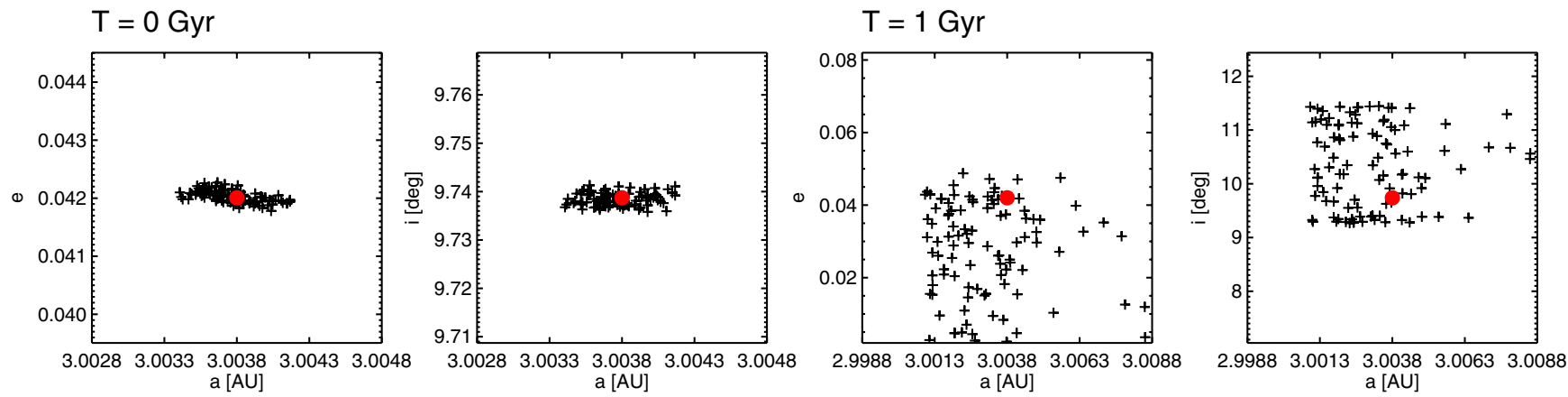

Figure 5. Dynamical evolution of 100 clones (black crosses) that started with orbits within the quoted $1 \sigma$ uncertainties of the orbit of $\mathrm{P} / 2012 \mathrm{~F} 5$ (filled red circle). After precessing the orbit for $1 \mathrm{Gyr}$, the particles have dispersed in orbital space but not significantly. The object P/2012 F5 can thus be considered a dynamically stable main belt asteroid, and not an interloper from the outer solar system.

(A color version of this figure is available in the online journal.)

nature of the coma, the radius of the asteroid is probably of the order of or less than $1 \mathrm{~km}$.

We also use the non-detection of P/2012 F5 in the WISE data to set an upper limit on the size of a bare nucleus that could have been present during 2010 September. The upper limit on the flux from the W3 stacked image and the NEATM model (Harris 1998; Delbó et al. 2003; Mainzer et al. 2011b) with a fixed beaming $(\eta)$ parameter of 0.8 (Lebofsky et al. 1986) and an assumed optical albedo of $0.04 \pm 0.02$ yielded a radius of $<2.9 \mathrm{~km}$, for a $1 \sigma$ confidence interval.

The upper limits on the size of P/2012 F5 are consistent with reported sizes of other AMBOs, which range in radius from $120 \mathrm{~m}$ to $113 \mathrm{~km}$, with a median radius of $2.2 \mathrm{~km}$. It should be noted that all but one AMBOs have reported radii under $5 \mathrm{~km}$.

To determine whether P/2012 F5 would have been observed by WISE if it displayed a similar brightness and morphology to its appearance in the Palomar images, we compare the lower limit on absolute magnitude, $H_{R, \text { WISE }} \gtrsim 15.2$, to that of $\mathrm{P} / 2012$ F5 during the Palomar observations. We use a $11^{\prime \prime} .9$ radius aperture (projected distance $1.8 \times 10^{4} \mathrm{~km}$ ) to match the physical distance subtended by the 9 .' 0 radius aperture used with the WISE data. Centering this aperture on the condensation observed in 2012 March, we find an apparent $R$-band magnitude of $18.67 \pm 0.02 \mathrm{mag}$, corresponding to an absolute magnitude of $14.37 \pm 0.02 \mathrm{mag}$. This is $\sim 0.8 \mathrm{mag}$ brighter than the limit set by WISE, suggesting that $\mathrm{P} / 2012 \mathrm{~F} 5$ would have been detectable by WISE in 2010 September, had it displayed 2012 levels of activity. We can thus say that the object must have had a different morphology when observed in 2010 and was possibly a bare asteroid.

We use the quantity $A f \rho$ as a measure of dust production as initially defined in A'Hearn et al. (1984) and further discussed in A'Hearn et al. (1995). We adapt the initial formulation given in A'Hearn et al. (1984) to give

$$
A f \rho=\rho\left(\frac{2 \Delta r_{H}}{a \rho}\right)^{2} 10^{0.4\left(m_{\odot \lambda}-m\right)},
$$

where $A$ is the geometric albedo, $f$ is a filling factor of the grains in the field of view, $\rho$ is the projected size of the aperture $(\mathrm{cm})$, $\Delta$ is the geocentric distance $(\mathrm{cm})$, and $r_{H}$ is the heliocentric distance (AU). $m$ is the phase-corrected magnitude of the material, while $m_{\odot \lambda}$ retains its previous definition. The model assumes that the coma follows simple radial outflow model, which is insufficient to explain the observed morphology of $\mathrm{P} / 2012 \mathrm{~F} 5$. We thus restrict ourselves to using photometric results from small apertures $\left(<3^{\prime \prime}\right)$ when calculating A $f \rho$. Since the coma does not follow the assumed radial model where $N(\rho)$ $\propto \rho$, where $N(\rho)$ is the number of dust grains within an aperture of size $\rho$, we find that the calculated values of $A f \rho$ are not independent of aperture size. We find that the value of $A f \rho$ peaks at $\rho \sim$ the FWHM of the image, and subsequently decreases by $\sim 1 \mathrm{~cm}$ for every $1^{\prime \prime}$ increase in aperture radius. For an aperture of radius 2 .' 1 (a projected distance of $3260 \mathrm{~km}$ ), Af $\rho=11.0 \mathrm{~cm}$ and $11.1 \mathrm{~cm}$ in $B$ and $R$ bands, respectively. The uncertainties are dominated by the morphological divergence of the data from the model of simple, symmetric outflow and are significant enough that the results from the different filters are consistent with each other. This suggests that the grains are gray in color.

Equation (4) can also be used to estimate the geometric crosssection of material within the trail if the apparent magnitudes of the trail are substituted for $m_{\lambda}$. We find $\sigma=3.6 \times 10^{8} \mathrm{~m}^{2}$ and $4.1 \times 10^{8} \mathrm{~m}^{2}$ for the $B$ - and $R$-band magnitudes, respectively. As discussed in Section 4.1, the minimum particle size within the trail is $\sim 20 \mu \mathrm{m}$, while the largest dust grains close to the nucleus may be $>\mathrm{cm}$-sized. By making the simplifying assumption that the bulk of the mass of the trail resides in spherical grains with radii of $100 \mu \mathrm{m}$ and bulk densities of $1000 \mathrm{~kg} \mathrm{~m}^{-3}$, we find that the mass of material within our apertures is $\sim 5 \times 10^{7} \mathrm{~kg}$. Taking the radius of $\mathrm{P} / 2012 \mathrm{~F} 5$ to be $\sim 1 \mathrm{~km}$ with a bulk density of $1000 \mathrm{~kg} \mathrm{~m}^{-3}$, the material in the trail amounts to $\sim 0.01 \%$ of the asteroid by mass.

\subsection{Dynamical Stability}

We next seek to constrain the origin of P/2012 F5 to test whether it likely to have originated in the main belt, or to have been recently inserted from elsewhere in the solar system. Using numerical simulations to model test particle evolution, we have investigated the stability of 100 clones of P/2012 F5 on the Gyr-timescale. Clone starting positions were randomly assigned according to a Gaussian distribution with an FWHM equal to the $1 \sigma$ uncertainties in the osculating elements of P/2012 F5 given by the JPL HORIZONS System. We integrated the orbits of each clone for 1 Gyr using the SWIFT_RMSVY symplectic integrator (Levison \& Duncan 1994; Brož 2006) with a 25 day timestep, and including the Venus, Earth, Mars, Jupiter, Saturn, Uranus, and Neptune as massive bodies in addition to the Sun. We ignore non-gravitational effects for the purpose of this simulation as they are under-constrained. No clones were lost from the system during the simulation and the orbital evolution of the clones is negligible over significant periods of time (Figure 5), indicating that $\mathrm{P} / 2012$ F5 is dynamically stable and is not likely to have been recently implanted in the Main Belt. 


\section{DISCUSSION}

Dynamical modeling of the ejecta of P/2012 F5 suggests that the activity was driven by a one-time event, approximately 260 days before our observations. This rules out continuous sublimation over a period of months, though a short, intense burst of sublimating material over a period of days could recreate the morphology observed. Considering the possibility that the activity is driven by the sublimation of sub-surface volatiles, we examine the orbital location of P/2012 F5 during the WISE and Palomar observations. P/2012 F5 was at a heliocentric distance of $2.90 \mathrm{AU}$ and six month post-perihelion during the WISE observations, which revealed that the object was not displaying the activity observed at its discovery in early 2012. Strangely, P/2012 F5 was discovered only months before reaching aphelion, at a time when its surface temperature is decreasing as its heliocentric distance increases. However, the eccentricity of $\mathrm{P} / 2012 \mathrm{~F} 5$ is only 0.04 , corresponding to only $\mathrm{a} \sim 6 \mathrm{~K}$ difference in surface temperature between perihelion and aphelion (assuming it absorbs and emits as an isothermal blackbody with an albedo of 0.04). We therefore do not expect $\mathrm{P} / 2012 \mathrm{~F} 5$ to be active only near perihelion, if the activity is driven by a temperature-dependent process, such as sublimation. It is difficult to reconcile the short period of activity with sublimation when dust tails, trails, and/or comae observed around other AMBOs, such as 133P, 238P, and P/2010 R2, are consistent with prolonged generation over a period of several months or more (Hsieh et al. 2004, 2009b, 2012b; Moreno et al. 2011a). We note that if the estimated ejected mass lost through sublimation over a period of two weeks, the mass-loss rate would be $\sim 25 \mathrm{~kg} \mathrm{~s}^{-1}$, orders of magnitude higher than that observed for other AMBOs whose activity is suspected to be driven by sublimation (Hsieh et al. 2004, 2009b; Jewitt et al. 2009). Observations of repeated activity on P/2012 F5 would strengthen the argument for sublimation-driven activity, especially if subsequent mass loss occurred at a similar orbital position to the 2011 outburst studied here.

Smaller asteroids are subject to non-gravitational forces that can cause rapid rotational spin-up (Rubincam 2000). If the rotational velocity of material on the surface exceeds the escape velocity, material may be ejected (Jewitt 2012). The fate of ejecta is not well constrained, but work by Walsh et al. (2008) suggests that the material would be lifted into a low orbit where it may eventually accrete into a satellite. This is supported by observations of asteroid $1999 \mathrm{KW}$, which is rotating with a period close to the limit, has a binary companion and is presumed to be losing mass from its elongated equator (Ostro et al. 2006; Scheeres et al. 2006). Due to a lack of well-studied examples, it is unclear whether rotational breakup could recreate the morphology observed around P/2012 F5, or whether it would eject sufficient mass on a short timescale of just a few weeks. Future measurements of the rotation period and size of $\mathrm{P} / 2012$ F5 could assist with gauging the likelihood of this scenario by calculating whether the nucleus is rotating rapidly enough to eject material.

The results of the dust modeling are also consistent with an impact that occurred $\sim 260$ days prior to our observations. If the activity observed around P/2012 F5 is due to a collision with a smaller asteroid, the mass of observed ejecta can be used to set an upper limit on the size of a possible impactor. A mass of $5 \times 10^{7} \mathrm{~kg}$, as calculated in Section 4.2, would correspond to a sphere of radius $20 \mathrm{~m}$, assuming a bulk density of $1000 \mathrm{~kg} \mathrm{~m}^{-3}$. As Jewitt et al. (2010) note, the majority of the mass lost from a small body during an impact is from the primary body, rather than the impactor. We thus conclude that the radius of an impactor would have been of the order of meters in size. The ejecta mass is comparable to estimates of the ejected mass around P/2010 A2 $\left(3.7 \times 10^{7} \mathrm{~kg}\right.$, Snodgrass et al. $2010 ; 5 \times 10^{7} \mathrm{~kg}$, Moreno et al. 2010; 6-60 × 10 $0^{7} \mathrm{~kg}$, Jewitt et al. 2010) and 596 (Scheila) $\left(3 \times 10^{7} \mathrm{~kg}\right.$, Hsieh et al. 2012a; $4 \times 10^{7} \mathrm{~kg}$, Jewitt et al. $2011 ; 1.5-4.9 \times 10^{8} \mathrm{~kg}$, Ishiguro et al. $2011 ; 6 \times 10^{8} \mathrm{~kg}$, Bodewits et al. 2011; $2 \times 10^{10} \mathrm{~kg}$, Moreno et al. 2011b), both of which are suspected to have undergone collisions in the main belt. With so few examples, it is difficult to know if the similarity of the ejecta masses is coincidence or intrinsic to the nature of main belt asteroidal collisions.

\section{SUMMARY}

We have used optical and infrared data to characterize P/2012 F5, which appears to be a main belt asteroid undergoing mass loss as a result of an impact with a smaller body.

1. A $1 \sigma$ upper limit on the radius of the nucleus is set at $2.9 \mathrm{~km}$ using WISE observations from 2010. A more sensitive upper limit of $2.1 \mathrm{~km}$ is set using $R$-band images from Palomar observatory. The cross-section of material observed near the nucleus in 2012 is above the detection limit set by the WISE data, suggesting that the asteroid was either inactive or weakly active during 2010 September.

2. Activity ejected $\sim 3 \times 10^{8} \mathrm{~m}^{2}$ of material into the trail, spanning $>7^{\prime}\left(6.5 \times 10^{5} \mathrm{~km}\right.$ projected distance $)$ in space. The minimum size of particles in the trail was $\sim 20 \mu \mathrm{m}$.

3. We find that syndynes do not fit the observed morphology, arguing against a period of continuous mass loss from the nucleus. Instead, a single event on UT 2011 July $7 \pm 20$ days can explain the observed shape of the trail.

4. Results from a symplectic integrator show the object to be dynamically stable and resident in the outer main belt on long timescales, suggesting P/2012 F5 is native to the main belt.

5. The observed behavior can be explained by a collision with a meter-sized impactor. Further observations may reveal morphological evolution in the trail that may shed more light on the cause of the activity.

This publication makes use of data products from the Widefield Infrared Survey Explorer, which is a joint project of the University of California, Los Angeles, and the Jet Propulsion Laboratory/California Institute of Technology, funded by the National Aeronautics and Space Administration. This work was based on observations obtained at the Hale Telescope, Palomar Observatory as part of a continuing collaboration between the California Institute of Technology, NASA/JPL, and Cornell University. The authors thank Henry Hsieh for bringing the discovery of P/2012 F5 to their attention. They also thank Kevin Rykoski, Carolyn Heffner, and Jean Mueller for assistance with the observations at Palomar Observatory. R.S. and J.M. acknowledge support from the NASA Postdoctoral Fellowship Program. E.K. was supported by the JPL Graduate Fellowship Program. This research was funded in part by a grant from NASA through the Near Earth Object observing programs, for the NEOWISE project. The supercomputer used in this investigation was provided by funding from the JPL Office of the Chief Information Officer. 


\section{REFERENCES}

A’Hearn, M. F., Millis, R. L., Schleicher, D. G., Osip, D. J., \& Birch, P. V. 1995, Icarus, 118, 223

Ahearn, M. F., Schleicher, D. G., Millis, R. L., Feldman, P. D., \& Thompson, D. T. 1984, AJ, 89, 579

Bauer, J. M., Mainzer, A. K., Grav, T., et al. 2012, ApJ, 747, 49

Belskaya, I. N., \& Shevchenko, V. G. 2000, Icarus, 147, 94

Bodewits, D., Kelley, M. S., Li, J.-Y., et al. 2011, ApJ, 733, L3

Brož, M. 2006, PhD thesis, Charles Univ.

Burns, J. A., Lamy, P. L., \& Soter, S. 1979, Icarus, 40, 1

Cutri, R. M., Wright, E. L., Conrow, T., et al. 2011, Explanatory Supplement to the WISE Preliminary Data Release Products, Technical Report

Delbó, M., Harris, A. W., Binzel, R. P., Pravec, P., \& Davies, J. K. 2003, Icarus, 166,116

Elst, E. W., Pizarro, O., Pollas, C., et al. 1996, IAU Circ., 6456, 1

Finson, M. J., \& Probstein, R. F. 1968, ApJ, 154, 327

Gibbs, A. R., Sato, H., Ryan, W. H., et al. 2012, CBET, 3069, 1

Haghighipour, N. 2009, Meteorit. Planet. Sci., 44, 1863

Harris, A. W. 1998, Icarus, 131, 291

Hsieh, H. H., \& Jewitt, D. 2006, Science, 312, 561

Hsieh, H. H., Jewitt, D. C., \& Fernández, Y. R. 2004, AJ, 127, 2997

Hsieh, H. H., Jewitt, D., \& Fernández, Y. R. 2009a, ApJ, 694, L111

Hsieh, H. H., Jewitt, D., \& Ishiguro, M. 2009b, AJ, 137, 157

Hsieh, H. H., Jewitt, D., Lacerda, P., Lowry, S. C., \& Snodgrass, C. 2010, MNRAS, 403, 363

Hsieh, H. H., Meech, K. J., \& Pittichová, J. 2011, ApJ, 736, L18

Hsieh, H. H., Yang, B., \& Haghighipour, N. 2012a, ApJ, 744, 9

Hsieh, H. H., Yang, B., Haghighipour, N., et al. 2012b, AJ, 143, 104

Ishiguro, M., Hanayama, H., Hasegawa, S., et al. 2011, ApJ, 740, L11

Jewitt, D. 1991, in IAU Colloq. 116: Comets in the Post-Halley Era, ed. R. L. Newburn, Jr., M. Neugebauer, \& J. Rahe (Astrophysics and Space Science Library, Vol. 167; Cambridge: Cambridge Univ. Press), 19

Jewitt, D. 2012, AJ, 143, 66

Jewitt, D., \& Meech, K. J. 1986, ApJ, 310, 937

Jewitt, D., Weaver, H., Agarwal, J., Mutchler, M., \& Drahus, M. 2010, Nature, 467,817
Jewitt, D., Weaver, H., Mutchler, M., Larson, S., \& Agarwal, J. 2011, ApJ 733, L4

Jewitt, D., Yang, B., \& Haghighipour, N. 2009, AJ, 137, 4313

Kolokolova, L., Hanner, M. S., Levasseur-Regourd, A.-C., \& Gustafson, B. Å S. 2004, in Physical Properties of Cometary Dust from Light Scattering and Thermal Emission, ed. M. C. Festou, H. U. Keller, \& H. A. Weaver (Tucson, AZ: Univ. of Arizona Press), 577

Lamy, P. L., Toth, I., Fernandez, Y. R., \& Weaver, H. A. 2004, in The Sizes, Shapes, Albedos, and Colors of Cometary Nuclei, ed. M. C. Festou, H. U. Keller, \& H. A. Weaver (Tucson, AZ: Univ. of Arizona Press), 223

Landolt, A. U. 1992, AJ, 104, 340

Lebofsky, L. A., Sykes, M. V., Tedesco, E. F., et al. 1986, Icarus, 68, 239

Levison, H. F., \& Duncan, M. J. 1994, Icarus, 108, 18

Licandro, J., \& Campins, H. 2010, in IAU Symp. 263, Icy Bodies of the Solar System, ed. J. A. Fernández, D. Lazzaro, D. Prialnik, \& R. Schulz (Cambridge: Cambridge Univ. Press), 215

Licandro, J., Campins, H., Tozzi, G. P., et al. 2011, A\&A, 532, A65

Lisse, C. M., A’Hearn, M. F., Hauser, M. G., et al. 1998, ApJ, 496, 971

Mainzer, A., Bauer, J., Grav, T., et al. 2011a, ApJ, 731, 53

Mainzer, A., Grav, T., Masiero, J., et al. 2011b, ApJ, 736, 100

Masci, F. J., \& Fowler, J. W. 2009, in ASP Conf. Ser. 411, Astronomical Data Analysis Software and Systems XVIII, ed. D. A. Bohlender, D. Durand, \& P. Dowler (San Francisco, CA: ASP), 67

Masiero, J. R., Mainzer, A. K., Grav, T., et al. 2011, ApJ, 741, 68

Moreno, F., Lara, L. M., Licandro, J., et al. 2011a, ApJ, 738, L16

Moreno, F., Licandro, J., Ortiz, J. L., et al. 2011b, ApJ, 738, 130

Moreno, F., Licandro, J., Tozzi, G.-P., et al. 2010, ApJ, 718, L132

Ostro, S. J., Margot, J.-L., Benner, L. A. M., et al. 2006, Science, 314, 1276

Rubincam, D. P. 2000, Icarus, 148, 2

Scheeres, D. J., Fahnestock, E. G., Ostro, S. J., et al. 2006, Science, 314, 1280 Schorghofer, N. 2008, ApJ, 682, 697

Simcoe, R. A., Metzger, M. R., Small, T. A., \& Araya, G. 2000, BAAS, 32, 758

Snodgrass, C., Tubiana, C., Vincent, J.-B., et al. 2010, Nature, 467, 814

Walsh, K. J., Richardson, D. C., \& Michel, P. 2008, Nature, 454, 188

Wright, E. L., Eisenhardt, P. R. M., Mainzer, A. K., et al. 2010, AJ, 140, 1868 